Aims.

- For $100 \%$ of patients admitted $\mathrm{OOH}$ (Friday $5 \mathrm{pm}$ - Sunday 9am) to have a multi-disciplinary review of their treatment

- For $100 \%$ of patients with deteriorating physical and mental health to be discussed

- To improve multi-disciplinary team (MDT) morale, working relationships and team cohesiveness $\mathrm{OOH}$

Background. In most specialties, the standard of best practice is that patients admitted to the ward out of hours $(\mathrm{OOH})$ receive a senior review over the weekend. However this does not usually take place in Psychiatry, and patients routinely wait until Monday to be seen by the ward team. This has been highlighted as problematic in cases where patients are agitated and not receiving any treatment for $>24$ hours.

We trialled a weekend teleconference safety huddle in Lewisham involving the on call consultant, registrar ( $\mathrm{SpR}$ ), core trainee (CT) and duty senior nurse (DSN).

Method. The weekend huddles were through a teleconference line, with participants dialling in at 9.45am.

Issues discussed:

1) Management plan for newly admitted patients $\mathrm{OOH}$.

2) Plan for patients with deteriorating mental health or escalating level of aggression.

3) Plan for patients with deteriorating physical health.

Feedback was collected from CTs, SpRs and consultants focusing on whether the huddle made any difference to the speed of care, cohesiveness of the $\mathrm{OOH}$ team, and whether it was generally helpful or not.

Result. 54\% of CTs $(n=11)$ felt that patients admitted OOH had an MDT review, and $90 \%$ felt that patients with deteriorating mental and physical health were discussed and a plan put in place. $80 \%$ of CTs, $63 \%$ of SpRs $(n=8)$ and $67 \%$ of consultants $(n=6)$ agreed it improved team cohesiveness. 90\% of DSNs felt safer and more supported in decision-making $\mathrm{OOH}$.

$80 \%$ of CTs, $63 \%$ of SpRs and $83 \%$ of consultants found weekend huddles helpful.

Data were also collected on violent incidents $\mathrm{OOH}$, and there was a slight reduction in the number of violent incidents in the weeks following introduction of the huddle.

Conclusion. Introducing safety huddles in Lewisham has facilitated the prompt discussion of the management of patients admitted $\mathrm{OOH}$, and of those with deteriorating mental and physical health. It has also fostered a greater sense of cohesiveness in the MDT team.

In light of this feedback, safety huddles have now been established as part of the weekend schedule in Lewisham, and are being rolled out to other boroughs within SLaM.

\section{Trust-wide improvement and standardisation of the medical handover}

Kabir Yisa*, Roshelle Ramkisson and Anoop Mohan

Pennine Care Foundation Trust

${ }^{*}$ Corresponding author.

doi: 10.1192/bjo.2021.613

Aims. Primary: To improve the quality of medical handover by increasing trust-wide completion rate for agreed quality standards from baseline to $80 \%$ by July 2019

To standardise the medical handover across all 5 boroughs within the trust by July 2019
Secondary: To separately assess the completion rate for new patient checks in relation to baseline

Background. Not only is there a recognised variation in the medical handover across Pennine care foundation trust's (PCFT) 5 boroughs (Tameside, Rochdale, Bury, Oldham and Stockport), but there also appears to be diminished adherence to quality standards to a varying extent. This was shown to result from a combination of human factors and process errors consequently leading to near-miss clinical incidents. This project was therefore designed to highlight and address these issues in order to promote patient safety.

Method. Having identified the problems and agreed on quality standards, baseline data were gathered through May 2019 with the primary outcome measure being the percentage completion rate for quality standards across all handovers. Changes were made to the handover document in June 2019 followed by introduction of a new handover document and post-intervention data collection through July 2019. Doctors' satisfaction with the new handover document using a typical 5-level Likert scale was measured using a trust-wide online survey.

Result. Our results showed a considerable Trust-wide improvement in the completion rate for agreed quality standards from $54 \%$ at pre-intervention to $77 \%$ at post-intervention with all 5 boroughs demonstrating improvement to varying extents. This is noted to be reasonably close to the project aim of improving the completion rate from baseline to $80 \%$. With regards to new patient checks, there was a marginal Trust-wide increase in completion rate from $53 \%$ at pre-intervention to $56 \%$ at postintervention. Additionally, the trust-wide online survey measuring doctors' satisfaction with the new handover revealed a majority $(82 \%)$, either strongly agreed $(27.3 \%)$, or agreed $(54.6 \%)$, that the new handover document has improved the overall quality of handovers.

Conclusion. Trust-wide standardisation of the handover has now presented the opportunity to benchmark boroughs within PCFT against one another in terms of adherence to quality standards and alignment with best practice relating to patient safety. While substantial improvement may have been made in the overall quality of handovers across the trust, the inter-borough disparity in the extent of improvement identified gaps for future follow-up or related projects.

\section{Research}

The impact of COVID-19 restrictions on the presentation of self-injury: experience at a tertiary centre

Tobias Adams ${ }^{1 *}$, A Arnaout ${ }^{2}$, S Bickerton ${ }^{2}, \mathrm{~L} \mathrm{Li}^{1}$, AWN Reid ${ }^{2}$ and SH Leow ${ }^{1}$

${ }^{1}$ Cambridge University Hospitals NHS Foundation Trust, University of Cambridge and ${ }^{2}$ Cambridge University Hospitals NHS Foundation Trust

${ }^{\star}$ Corresponding author.

doi: 10.1192/bjo.2021.614

Aims. The national lockdowns due to the COVID-19 pandemic, have had a considerable effect on mental health, with reduced access to social interaction through work and leisure activities, and increased barriers to community mental health services.

This study aims to evaluate how the presentation of patients with self-injury has changed during the first national U.K. lockdown, at a Plastic Surgery Tertiary Referral Centre in the East of England.

Method. Retrospectively recorded data from 23 March 2020 to 31 December 2020, spanning the duration of the first two lockdowns 
in the UK, were compared to the same period in 2019. The demographics of patients, along with the nature, severity and outcomes of the self-injury were recorded and compared.

Result. The number of patients referred for self-injury reduced by 22.9\% during lockdown (2020: $\mathrm{N}=42 / 109,2019$ : 67/109)

The most common attendance route was via ambulance during lockdown (2020:40.5\% 2019: 31.3\%) whilst the most common attendance route being via the front door in 2019 (2019: 35.8\%, 2020: $26.2 \%)$

The number of new presentations with no prior history of selfinjury was higher in lockdown $38.1 \%$ ) compared to $201926.9 \%$.

The lockdown cohort had a smaller proportion of patients presenting with complications (2020: $9.5 \%$ vs 2019: 17.9\%), less readmitted (2020: $11.9 \%$ vs 2019: $23.9 \%$ ). Similar re-attendance rate (2020: $40.3 \%$ vs 2019: 38.1\%) and re-intervention (2020: $13.4 \%$ vs $2019: 14.3 \%)$.

A greater proportion in 2020 met the threshold for inpatient psychiatry input (2020: $52.4 \%$ vs 2019: $41.8 \%$ ).

During the lockdown, a higher percentage of flexor tendon injuries involved multiple tendons $(60.0 \%$ vs $52.2 \%)$. A higher percentage of extensor tendon injuries (14.3\% vs $7.4 \%)$, and a greater proportion of these also involved multiple tendons $(66.7 \%$ vs $40.0 \%)$. More self-injuries were complicated by fractures $(7.1 \%$ vs $4.5 \%)$ and more required soft tissue reconstruction (11.9\% vs $3.0 \%)$. Conclusion. Despite fewer patients presenting with self-injury during the 2020 lockdown, the injuries were more severe. Many of which had multiple structural injuries, and some with lifechanging injuries, this is in line with our clinical observations.

During lockdown there was a higher proportion of first-time presentations without a history of self-injury and an increased need for inpatient psychiatry input. This may reflect the impact on mental health as a result of restricted social interactions.

These findings demonstrate the impact of lockdown on mental-health and may help inform medical services of potential changes in the presentations in future national social restrictions.

\section{Growing pains: a scoping literature review of how} perinatal psychiatry was impacted by COVID-19

Stephanie Adeyemi

East and North Hertfordshire NHS Trust

doi: 10.1192/bjo.2021.615

Aims. This scoping review aims to assess the impact of COVID-19 on the field of Perinatal Psychiatry and identify any innovations made as a result of this.

Background. The World Health Organisation declared the COVID-19 outbreak a global pandemic on March 11th 2020. This pandemic has transformed the way in which Perinatal Psychiatric services are delivered in the United Kingdom and countries across the globe acting as a catalyst for innovation.

Method. The databases searched for peer reviewed literature written since December 2019 were: PsychINFO, MEDLINE, EMBASE, CINAHL and PUBMED. Search strategy key words were: COVID-19, SARS-CoV-2, perinatal psychiatry and maternal mental health. Arksey and O’Malley's framework was utilised. Data were collated and summarized thematically.

Result. 42 studies met the inclusion criteria. The aforementioned studies included data from over 60,000 women from the following countries: China, Italy, Netherlands, United States, United Kingdom, Brazil, India, Spain, Ireland, Norway, Switzerland, Iran, Japan and Nepal. Literature clearly indicates that during the pandemic there was an increase in depression and anxiety. Risk factors included: financial insecurity, disrupted antenatal care, isolation, poor physical health and domestic violence. Evidence also suggested COVID-19 stressors impacted feeding practices and infant development as cytokines pass from mother to baby.

Perinatal Psychiatry services have adopted social media apps to provide antenatal information, teleconsultations, smartphonebased cognitive-behavioral therapy (iCBT) programs and increased utilisation of screening tools such as the Pandemic-Related Pregnancy Stress Scale (PREPS), the Edinburgh Postnatal Depression Scale (EPDS) and the Postpartum Specific Anxiety Scale.

Conclusion. Whilst this review features literature centred on women from across the globe African women are underrepresented. This should be addressed in future studies. This review shows that the COVID-19 pandemic has impacted maternal mental health and acted as a catalyst for innovation. It is essential that efforts are made to support women during pregnancy and the perinatal period now more than ever.

\section{Skin Deep? : A scoping literature review of the psychological impact of Acne Vulgaris on patients and the role of the Psychiatrist}

Stephanie Adeyemi

East and North Hertfordshire NHS Trust

doi: 10.1192/bjo.2021.616

Aims. This literature review aims to assess existing scientific literature on the psychological impact of Acne Vulgaris on adolescents and adults and the role that Psychiatrists can play in supporting these patients. The hypothesis of this literature review is that all patients with Acne Vulgaris should have their quality of life assessed in order to identify those who require additional support.

Background. The link between Psychiatry and Dermatology is becoming increasingly recognised. Resources on the British Association of Dermatologists' website are often distributed to patients by Dermatologists in order to assess the impact that a skin condition has had on a patient's life. Acne Vulgaris is a psychophysiological skin condition that impacts up to $95 \%$ of people to some extent from the ages of 11 to 30 years old. Due to its prevalence it is essential that the psychological burden of Acne Vulgaris on patients is understood.

Method. Literature written since 2011 was searched identified from: PsychINFO, MEDLINE, EMBASE, CINAHL and PUBMED. The search strategy key words were: acne vulgaris, mental health, psychiatry, anxiety and depression. Arksey and O'Malley's framework was utilised to conduct a scoping literature review. Data were collated and summarized thematically.

Result. A total of 72 studies were included representing over 14,000 adults and adolescents with Acne Vulgaris from the following countries: Egypt, Nigeria, Turkey, India, Lithuania, UK, USA, Iran, Pakistan and Spain.

Screening tools such as the Global Acne Grading System (GAGS), The Acne Quality of Life Scale (AQLS), the Cardiff Acne Disability Index (CADI), and the State Trait Anxiety Index STAI (Y-1) form were utilised in order to identify the impact of Acne Vulgaris on patients' quality of life and mental health. The data clearly showed the significant psychological burden that patients with Acne Vulgaris can experience. There was a clear trend of low self-esteem, lack of self-confidence, social withdrawal, depression (ranging from $23.1 \%$ to $62 \%$ of study participants), anxiety (ranging from $38.4 \%$ to $51 \%$ ) and even suicidal ideation (ranging from $12.9 \%$ to $20.1 \%$ ). Literature also 